\title{
Saturation hysteresis effects on the seismic signatures of partially saturated heterogeneous porous rocks
}

\author{
Santiago G. Solazzi ${ }^{1,2}$, Luis Guarracino ${ }^{2}$, J. Germán Rubino ${ }^{3}$, and Klaus \\ Holliger $^{1,4}$ \\ ${ }^{1}$ Applied and Environmental Geophysics Group, Institute of Earth Sciences, University of Lausanne, \\ Lausanne, Switzerland. \\ ${ }^{2}$ CONICET, Facultad de Ciencias Astronómicas y Geofísicas, Universidad Nacional de La Plata, La \\ Plata, Argentina. \\ ${ }^{3}$ CONICET, Centro Atómico Bariloche - CNEA, San Carlos de Bariloche, Argentina. \\ ${ }^{4}$ School of Earth Sciences, Zhejiang University, Hangzhou, China.
}

Key Points:

- We present a novel model that allows to include the effects of saturation hysteresis on seismic attenuation and phase velocity dispersion.

- We reproduce key features of the saturation fields and of the seismic signatures observed during drainage and imbibition experiments.

- Results show that the pore-scale characteristics can greatly influence the hysteresis effects on the seismic signatures.

Published (2019) American Geophysical Union. Further reproduction or electronic distribution is not permitted. Not subject to U.S. copyright. The AGU published article can be found in: https://doi.org/10.1029/2019JB017726

Corresponding author: Santiago G. Solazzi, Santiago.Solazzi@unil.ch 


\section{Abstract}

Experimental evidence indicates that the spatial distribution of immiscible pore fluids in partially saturated media depends on the flow history and, thus, exhibits hysteresis effects. To date, most works concerned with modelling the effective seismic properties of partially saturated rocks either disregard these effects or account for them employing oversimplified approaches. This, in turn, can lead to erroneous interpretations of the corresponding seismic signatures. In this work, we present a novel methodology that allows to compute hysteresis effects on seismic attenuation and dispersion due to mesoscopic wave-induced fluid flow (WIFF) in realistic scenarios. For this purpose, we first employ a constitutive model that considers a porous medium locally as a bundle of constrictive capillary tubes with a fractal pore-size distribution, which allows to estimate local hydraulic properties and capillary pressure-saturation hysteretic relationships in a heterogeneous rock sample. Then, we use a numerical upscaling procedure based on Biot's poroelasticity theory to compute seismic attenuation and velocity dispersion curves during drainage and imbibition cycles. By combining these procedures, we are able to model, for the first time, key features of the saturation field and of the seismic signatures commonly observed in the laboratory during drainage and imbibition experiments. Our results also show that the pore-scale characteristics of a given porous medium, such as the pore-throat geometry, can greatly influence the hysteresis effects on the seismic signatures.

\section{Introduction}

Partially saturated environments are of preeminent importance in many scientific and applied scenarios, such as, groundwater management and remediation, exploration and production of hydrocarbons, and $\mathrm{CO}_{2}$ geosequestration. Partially saturated geological formations are commonly modelled as porous media whose pore space is occupied simultaneously by two immiscible and mobile fluid phases (e.g., Bear, 1972). These fluid phases are referred to as wetting and non-wetting in relation to their capacity to wet the pore walls. Interestingly, the spatial distribution of the pore fluids throughout a porous medium is determined by the heterogeneities of the rock frame, the properties of the pore fluids, and by the flow history (e.g., Shi et al., 2011; Alemu et al., 2013). In this context, a fundamental aspect to account for is the irreversibility of multiphase flow dynamics, that is, the hysteresis of this physical process. 
At the microscopic scale, hysteresis is mainly considered to be caused by contact angle effects (Juanes et al., 2006) and by irregularities in the cross-sections of the pores that act as "capillary barriers" to the flow of the non-wetting phase (e.g., Lenormand, 1990; Soldi et al., 2017). Hysteretic effects are usually defined in terms of the two extreme cases of immiscible displacement, namely, imbibition and drainage. Imbibition is a process where an invading wetting fluid phase displaces an already present non-wetting phase from the rock pores. Drainage is the inverse process, that is, a non-wetting phase displaces a wetting phase from the pore space. Employing computer-assisted tomography $(\mathrm{CT})$ scans, several experimental works show that drainage and imbibition processes generate fundamentally different saturation patterns for the same overall saturation state (e.g., Cadoret et al., 1998; Shi et al., 2011; Alemu et al., 2013). Therefore, hysteretic effects should be accounted for when trying to characterize the properties of partially saturated media through non-invasive geophysical methods.

The seismic method is arguably one of the most employed techniques to explore the subsurface (e.g., Kearey et al., 2013). Improving the current understanding of the properties of seismic waves traveling through partially saturated environments could allow to extract crucial information, such as permeability field and fluid distribution, from seismic data. One of the first experimental studies focusing on the impact of saturation hysteresis on seismic signatures was performed by Knight and Nolen-Hoeksema (1990). They observed that the relationship between seismic velocity and overall saturation differs when the saturation state of the probed rock sample is obtained through drainage or imbibition. Later, Yin et al. (1992) observed a similar behavior on attenuation curves and attributed their results to wave-induced fluid flow (WIFF) (e.g., Müller et al., 2010) taking place in the mesoscopic scale range, that is, at scales much larger than the pore scale but much smaller than the predominant seismic wavelength, between fully watersaturated regions and their partially saturated surroundings. A common feature of these experimental studies is that attenuation and phase velocity dispersion values are more pronounced during drainage than during imbibition. The works of Cadoret et al. (1995, 1998), which explored the behavior of seismic signatures for different frequencies and saturations in partially saturated limestones, shed some light on this particular subject. These authors employed CT scans to determine the air and water distribution associated with drainage and imbibition processes. They observed that drainage processes tend to generate non-uniform fluid distributions characterized by well-defined gas and water patches. 
Conversely, imbibition processes tend to produce more uniform fluid distributions with smoother transitions between the water-saturated patches and their surroundings. The more accentuated mechanical compressibility contrasts generated by drainage would therefore be expected to produce higher dissipation due to WIFF than those resulting from imbibition experiments. It is worth mentioning that not all the works studying the effects of hysteresis on the seismic signatures of porous rocks evidence such behavior (e.g., Nakagawa et al., 2013; Alemu et al., 2013; Zhang et al., 2015). Hence, the complexity of hysteresis processes should be further analyzed if we wish to discern the physical mechanisms that control the characteristics of the saturation distribution and of the associated seismic response.

To date, theoretical works accounting for hysteretic effects on the seismic signatures of partially saturated media rely on simplifying assumptions that limit a rigorous interpretation of the governing physical processes. Akbar et al. (1994) and Papageorgiou and Chapman (2015) modelled saturation hysteresis effects on squirt flow. Although the squirt flow models proposed by these works are fundamentally different, they both consider that the porous medium is composed by stiff pores and compliant "cracks" and use simple models to saturate these regions. Le Ravalec et al. (1996) proposed a model to account for the effects of hysteresis on seismic phase velocities due to mesoscopic WIFF and squirt flow. These authors consider partially saturated spherical patches to model mesoscopic WIFF effects and round pore and spheroidal crack geometries to model squirt flow effects. In this model, local saturation depends on the drainage or imbibition processes. It is important to remark here that all the above mentioned models assume that the rock samples are homogeneous with regard to porosity and permeability. However, experimental evidence shows that even clean and well-sorted sandstone samples tend to exhibit substantial fluctuations of their hydraulic properties (e.g., Krause et al., 2013; Li \& Benson, 2015). Without the existence of such heterogeneities to trap the pore fluids, mesoscopic scale fluid patches would migrate due to buoyant forces and diffuse due to the effects of capillary pressure gradients (e.g., Krevor et al., 2011). In this sense, laboratory measurements show conclusively that the fluid distribution is conditioned by the rock frame hydraulic properties (e.g., Shi et al., 2011; Alemu et al., 2013). In such context, Ba et al. (2015) proposed a double-porosity model, considering spherical patches and heterogeneous samples, in which hysteretic effects are included by assuming different saturation or desaturation scenarios. However, the considered fluid patches are not 
directly associated with changes in the hydraulic properties of the rock frame. Furthermore, the spherical patch geometry employed by Ba et al. (2015) and Le Ravalec et al. (1996) imposes an unrealistically sharp transition of physical properties between the mesoscopic patches and their surroundings, which has a strong impact on the seismic signatures (Rubino \& Holliger, 2012; Solazzi, 2018). Notably, the available evidence from laboratory experiments points to spatially continuous variations of the fluid distributions in partially saturated porous media (e.g., Toms-Stewart et al., 2009; Shi et al., 2011). To our knowledge, saturation hysteresis effects on mesoscopic WIFF have so far not been studied considering realistic spatially continuous saturation patterns governed by variations of the rock frame properties.

In this work, we present a novel model that allows to include the effects of saturation hysteresis on seismic attenuation and phase velocity dispersion due to mesoscopic WIFF in heterogeneous porous media. For this purpose, we employ a pore-scale model which considers the porous medium as a bundle of constrictive capillary tubes with a fractal pore size distribution (Soldi et al., 2017). This physically-based model has the advantage of providing closed analytical expressions for the porosity, the permeability, and the primary drainage and imbibition capillary pressure-saturation curves for a homogeneous porous medium. By assuming that the different regions of a heterogeneous rock sample are locally described by this constitutive model and considering a set of capillary equilibrium states, we obtain pore fluid distributions representative of both drainage and imbibition cycles. We then apply a numerical upscaling procedure based on Biot's theory of poroelasticity to compute seismic attenuation and dispersion curves due to WIFF produced by the heterogeneous fluid distribution. We explore the impact of saturation hysteresis on the fluid distribution and on the seismic signatures for different overall saturations and frequencies. Finally, we analyze the effects of the pore geometry on the hysteresis phenomenon. The proposed model permits to reproduce key features of the fluid distribution and of the seismic signatures observed in the laboratory during drainage and imbibition processes and, thus, allows for a better understanding of the WIFF phenomenon in partially saturated environments.

\section{Theoretical Background and Numerical Models}

In this section, we introduce the constitutive model of Soldi et al. (2017), which allows to obtain the porosity, the permeability, and the hysteretic capillary pressure-saturation 
curves of a porous medium characterized by a given pore space topology. Subsequently, we employ these relationships to determine the local hydraulic properties of a heterogeneous synthetic rock sample and, in particular, to generate saturation fields representative of drainage and imbibition processes. Finally, we present an upscaling procedure (Rubino et al., 2009) based on Biot's poroelasticity theory (Biot, 1941) to estimate the seismic attenuation and phase velocity dispersion of the numerical rock sample accounting for hysteresis effects.

\subsection{Hysteretic Model for Partially Saturated Rocks}

Capillary forces play a predominant role in the flow of immiscible fluid phases through porous formations. Interestingly, the distribution of immiscible fluid phases during capillarydriven flow is determined by mechanisms that take place at the pore scale (e.g., Lenormand et al., 1983). In this sense, microscopic processes provide the foundations for understanding and predicting two-phase flow at the field scale (e.g., Juanes et al., 2006).

At the macroscopic scale, the hysteresis process manifests itself through the dependence of the relative permeabilities and capillary pressures on the saturation history. Note that constitutive relationships, such as those of Brooks and Corey (1964) or van Genuchten (1980), have to be adapted to be history-dependent to account for this characteristic (e.g., Hogarth et al., 1988; Lenhard et al., 1991). Particularly, constitutive models based on capillary tubes have been proven to be useful to characterize porous media when describing hydrological processes and hydraulic properties for different granulometries (e.g., Tyler \& Wheatcraft, 1990; Yu et al., 2003; Guarracino et al., 2014; Xu, 2015). These models derive the hydraulic properties of a given porous medium considering that, in the presence of a fluid pressure gradient, flow channels are generated within the pore space. The characteristics of these channels are then modelled employing the capillary tube geometry considering different shapes and aperture distributions. If the rock is isotropic, the derived hydraulic properties are independent of the flow direction. In this context, Soldi et al. (2017) proposed a hysteretic constitutive model for partially saturated flow assuming that porous media can be conceptualized as a bundle of constrictive capillary tubes with a fractal distribution of the radii. Individual pores are modelled as cylindrical tubes of radius $r$ connected by periodical throats (Figure 1). Based on physical and geometrical concepts, closed-form equations for the porosity and permeability can be obtained by volume integration. Also, the chosen conceptualization of the pore geometry allows 


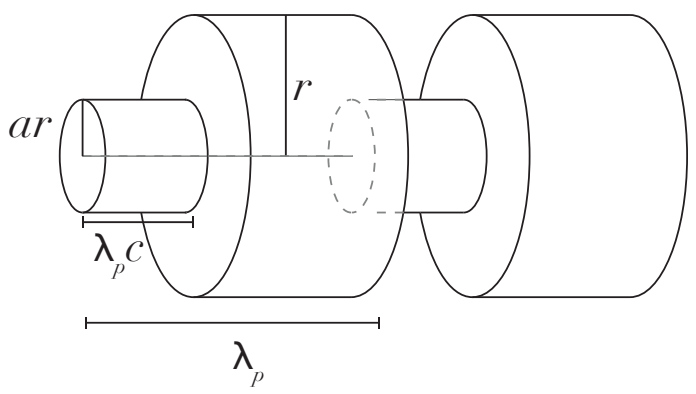

Figure 1. Pore geometry of a capillary tube of radius $r . \lambda_{p}$ is the period of the pore structure. The throats have radii and lengths given by $a \cdot r$ and $\lambda_{p} \cdot c$, respectively.

to model hysteresis due to "capillary barrier" effects in the capillary pressure-saturation functions. In this work, we use the model proposed by Soldi et al. (2017), whose characteristics are outlined below, to develop realistic partially saturated environments accounting for hysteresis effects.

Let us consider a representative elementary volume (REV) of a porous medium whose pore structure is represented by a bundle of constrictive tubes with varying radii $r$. Each constrictive tube is characterized by a spatial period $\lambda_{p}$, a radial factor $0<a \leq 1$, and a length factor $0 \leq c \leq 1$ (Figure 1). The radial factor $a$ represents the throatto-pore size ratio and the length factor $c$ represents the fraction of $\lambda_{p}$ with a narrow throat. The cumulative size distribution of the pores obeys a fractal law (e.g., Guarracino, 2007; Yu et al., 2003)

$$
N(r)=\left(\frac{r}{R}\right)^{-D}, \quad r_{\min } \leq r \leq r_{\max }
$$

where $R$ is the characteristic size of the REV, $1<D<2$ is the fractal dimension, and $r_{\min }$ and $r_{\max }$ are the minimum and maximum pore radii, respectively.

By means of volume integration, it is found that the porosity $\phi$ of the REV is given by (Soldi et al., 2017)

$$
\phi=\frac{f_{v} D}{R^{(2-D)}(2-D)}\left[r_{\max }^{(2-D)}-r_{\min }^{(2-D)}\right]
$$

where $f_{v}=a^{2} c+1-c$. The factor $f_{v}$ varies between 0 and 1 and quantifies the porosity reduction due to the constrictivity of pores. Also, by integrating the flow rate and 
employing Darcy's law, Soldi et al. (2017) inferred the effective permeability $\kappa$ as

$$
\kappa=\frac{f_{k} D}{8 R^{(2-D)}(4-D)}\left[r_{\max }^{(4-D)}-r_{\min }^{(4-D)}\right],
$$

where $f_{k}=a^{4} /\left[c+a^{4}(1-c)\right]$. The factor $f_{k}$ also varies between 0 and 1 and quantifies the permeability reduction due to the pore constrictivity.

As previously stated, the pore-scale geometry illustrated in Figure 1 permits to include hysteresis effects associated with the capillary pressure-saturation curve. Recall that, for a straight tube of radius $r_{p}$, the capillary pressure $p_{c}$ can be expressed as (Bear, 1972)

$$
p_{c}=\frac{2 \gamma \cos (\beta)}{r_{p}},
$$

where $\gamma$ is the interfacial tension between the two immiscible phases that occupy the pore space and $\beta$ the contact angle of the corresponding interface with the pore wall. Due to the varying aperture of the pores, drainage and imbibition processes exhibit distinct behaviors. In an imbibition process, capillary pressure drops as the porous rock is invaded by the wetting fluid. Following equation (4), smaller pores are wetted in the early stages of the process and larger pores follow. During a drainage process, capillary pressure increases as the pores are invaded by the non-wetting fluid. However, the process is conditioned by the throat size that connects the pores. Consequently, pores connected by thick throats are drained first and pores connected by narrow throats follow.

The main drainage capillary pressure-saturation curve is obtained by assuming that a pore becomes fully saturated by the non-wetting fluid if the radius of the pore throat $r_{t h}=a r$ is greater than the radius $r_{p}$ given by equation (4). Then, it is reasonable to conclude that pores with radii $r$ between $r_{\text {min }}$ and $r_{p} / a$ remain fully saturated by the wetting fluid. The closed-form analytical expression that relates the effective wetting fluid saturation and the capillary pressure for the drainage cycle $S_{e w}^{d}\left(p_{c}\right)$ is (Soldi et al., 2017)

$$
S_{e w}^{d}\left(p_{c}\right)=\left\{\begin{array}{lr}
1, & \text { if } p_{c} \leq \frac{p_{c, \min }}{a}, \\
\frac{\left(p_{c} a\right)^{(D-2)}-p_{c, \text { max }}^{(D-2)}}{p_{c, \text { min }}^{(D-2)}-p_{c, \text { max }}^{(D-2)}}, & \text { if } \frac{p_{c, \text { min }}}{a} \leq p_{c} \leq \frac{p_{c, \max }}{a}, \\
0, & \text { if } p_{c} \geq \frac{p_{c, \max }}{a},
\end{array}\right.
$$

where $p_{c, \text { min }}=2 \gamma \cos (\beta) / r_{\max }$ and $p_{c, \max }=2 \gamma \cos (\beta) / r_{\min }$ are are the minimum and maximum capillary pressures, respectively.

Similarly, the main imbibition capillary pressure-saturation curve can be obtained assuming that only the tubes with radius $r<r_{p}$ will be fully saturated by the wetting 


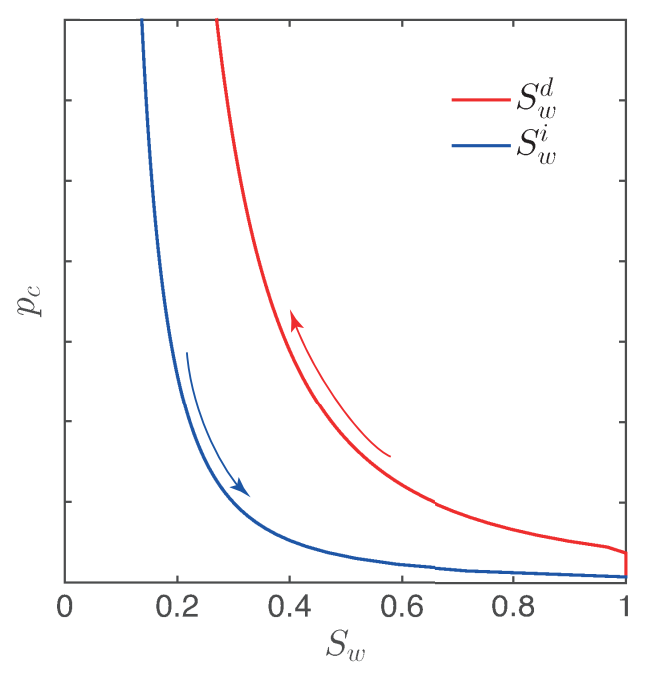

Figure 2. General behavior of the capillary pressure curves as a function of wetting phase saturation for drainage (red solid line) and imbibition (blue solid line) resulting from the hysteretic constitutive model.

fluid. Then, the effective wetting phase saturation for the main imbibition curve $S_{e w}^{i}\left(p_{c}\right)$ can be expressed as (Soldi et al., 2017)

$$
S_{e w}^{i}\left(p_{c}\right)=\left\{\begin{array}{lr}
1, & \text { if } p_{c} \leq p_{c, \min } \\
\frac{p_{c}^{(D-2)}-p_{c, \max }^{(D-2)},}{p_{c, \text { min }}^{(D-2)}-p_{c, \text { max }}^{(D-2)},} & \text { if } \quad p_{c, \min } \leq p_{c} \leq p_{c, \max } \\
0, & \text { if } p_{c} \geq p_{c, \max }
\end{array}\right.
$$

The saturation of the wetting phase can be obtained from equations (5) and (6) by means of $S_{w}^{q}=S_{e w}^{q}\left(1-S_{w r}\right)+S_{w r}$ with $q=i, d$, where $S_{w r}$ is the residual wetting phase saturation of the REV.

Figure 2 illustrates the general behavior of the main drainage (red solid curve) and imbibition (blue solid curve) capillary pressure curves as a function of wetting phase saturation resulting from equations (5) and (6). Due to the hysteretic nature of the proposed constitutive relationships, drainage and imbibition curves differ. Note that, for a given capillary pressure value, drainage curves are associated with higher saturation values than imbibition curves. It is important to remark that the hysteretic behavior described by equations (5) and (6) is conditioned by the radial factor $a$. That is, for $a=$ 1 drainage and imbibition capillary pressure-saturation curves are identical. 
The constitutive model presented in this section has the advantage of providing simple analytical expressions for porosity, permeability, and hysteretic capillary pressuresaturation functions for a homogeneous porous medium. We shall use these expressions to locally characterize a heterogeneous porous medium, assuming that each region of the rock sample is described by a particular set of pore geometry parameters $\left(r_{\max }, r_{\min }\right.$, $a, c, R$, and $D)$. Then, by assuming different stages of capillary pressure equilibrium at the sample's scale, we are able to compute heterogeneous saturation patterns which are representative of drainage and imbibition processes.

\subsection{Numerical Upscaling Procedure for Quantifying WIFF Effects}

Whenever a seismic wave propagates through a porous medium that contains mesoscopic heterogeneities, local gradients in the pore fluid pressure arise due to the uneven response of the different regions of the rock to the stresses associated with the passing wavefield (e.g., Pride, 2005). These pressure gradients induce viscous fluid flow and, thus, energy dissipation through internal friction. This mechanism, known as mesoscopic WIFF, can generate significant attenuation and velocity dispersion within the seismic exploration frequency band (Müller et al., 2010). A particularly interesting characteristic of the WIFF process is that it is sensitive to the hydraulic properties of the heterogeneous rock and to the geometrical characteristics of the pore fluid patterns (Rubino \& Holliger, 2012; Masson \& Pride, 2011). Consequently, hysteretic effects are expected to have a profound impact on seismic attenuation and phase velocity dispersion related to this mechanism.

In order to quantify WIFF effects produced by 2D heterogeneous partially saturated rocks, saturated following the procedure described in the previous subsection, we apply the numerical upscaling procedure proposed by Rubino et al. (2009). That is, we impose a homogeneous time-harmonic vertical solid displacement of the form $-\Delta u e^{i \omega t}$ along the top boundary of a bidimensional square representative sample of the explored formation, where $\omega$ is the angular frequency. In addition, no-flow conditions are imposed on all four boundaries and no tangential forces are applied. The solid is neither allowed to move vertically on the bottom boundary nor to have horizontal displacements on the lateral boundaries. The response of the sample subjected to this relaxation test is obtained by solving Biot's consolidation equations (Biot, 1941) under appropriate boundary conditions. Under the assumption that the volume-averaged response of the sample can be represented with an equivalent homogeneous viscoelastic solid, an equivalent 
complex-valued frequency-dependent plane wave modulus $M_{c}(\omega)$ is obtained. The inverse quality factor and phase velocity can be computed as (e.g., Borcherdt, 2009)

$$
\begin{gathered}
Q_{p}{ }^{-1}(\omega)=\frac{\Im\left\{M_{c}(\omega)\right\}}{\Re\left\{M_{c}(\omega)\right\}}, \\
V_{p}(\omega)=\left[\Re\left\{\sqrt{\frac{\left\langle\rho_{b}\right\rangle}{M_{c}(\omega)}}\right\}\right]^{-1},
\end{gathered}
$$

where $\left\langle\rho_{b}\right\rangle$ is the volume average of the bulk density of the aggregate and $\Re$ and $\Im$ denote the real and imaginary parts, respectively. The local bulk density is given by

$$
\rho_{b}=(1-\phi) \rho_{s}+\phi \rho_{f},
$$

where $\rho_{s}$ and $\rho_{f}$ are the densities of the solid grains and the fluid phase, respectively. Appendix A provides the details of this numerical upscaling procedure.

Please note that Biot's theory is based on the assumption of a single pore fluid phase. However, in a partially saturated medium, each cell of the numerical rock sample considered in the upscaling procedure may be saturated by both immiscible phases. Therefore, we locally employ an effective fluid phase when solving poroelastic equations A1 to A4. That is, at each computational cell we define an effective single phase fluid with properties determined by those of the individual fluid phases and weighted by their saturation values (Rubino \& Holliger, 2012). Then, the density of the effective fluid is given by

$$
\rho_{f}=S_{w} \rho_{w}+\left(1-S_{w}\right) \rho_{n},
$$

where $\rho_{w}$ and $\rho_{n}$ are the wetting and non-wetting phase densities, respectively.

As previously stated, the compressibility of the effective fluid is a crucial parameter in the WIFF process. Provided that we consider computational cells having sizes much smaller than the diffusion lengths associated with the WIFF process, the fluid pressure perturbations caused by the seismic wavefield have enough time to equilibrate within each computational cell. Hence, the fluid pressure within each cell is uniform and we can use Wood's law to obtain the bulk modulus of the effective fluid (Wood, 1955; Mavko et al., 2009; Rubino \& Holliger, 2012)

$$
\frac{1}{K_{f}}=\frac{\left(1-S_{w}\right)}{K_{n}}+\frac{S_{w}}{K_{w}},
$$

where $K_{n}$ and $K_{w}$ are the bulk moduli of the non-wetting and wetting phases, respectively. 
On the other hand, we use the relation of Teja and Rice (1981) to obtain the viscosity of the two-phase pore fluid mixture in each cell

$$
\eta_{f}=\eta_{n}\left(\frac{\eta_{w}}{\eta_{n}}\right)^{S_{w}}
$$

where $\eta_{w}$ and $\eta_{n}$ denote the viscosities of the wetting and non-wetting phases, respectively.

It is important to remark here that, even though we do consider the effects of capillary forces to determine the pore fluid distribution, capillary pressure is not accounted for when quantifying WIFF effects, as a single fluid with effective properties is considered in Biot's equations. Please also note that in this study we analyze hysteresis effects on WIFF at the mesoscopic scale and, thus, effects associated with fluid pressure diffusion at the pore scale are not accounted for in our model. Even though squirt flow effects are beyond the scope of this work, it is worthwhile to mention that they can indeed be modelled in conjunction with mesoscopic WIFF (e.g., Rubino et al., 2013). For this, both microscopic and mesoscopic WIFF models should be based on a unique and consistent pore scale conceptualization.

\section{Numerical Analysis}

\subsection{Heterogeneous Rock Sample and Physical Properties}

In the following, we explore the seismic response of a partially saturated porous medium during drainage and imbibition cycles. To do so, we analyze the behavior of a square 2D synthetic rock sample of 3-m side length with properties representative of a heterogeneous Fontainebleau sandstone (e.g., Bourbié \& Zinszner, 1985). We assume that the sample contains spatially continuous variations of the dry frame properties, which are parameterized as functions of the maximum pore radius. In particular, the spatial distribution of $r_{\max }$, shown in Figure 3a, is obtained by means of a stochastic procedure based on a von-Karman-type spectral density function (Tronicke \& Holliger, 2005). To this end we consider a stochastic process with a spatially isotropic correlation length of $25 \mathrm{~cm}$ and a Hurst number of 0.1. The minimum pore radius in each cell of the rock sample is considered to obey $r_{\min }=10^{-1} r_{\max }$. The resulting range of variations of both $r_{\max }$ and $r_{\min }$ is consistent with experimental measurements performed in Fontainebleau sandstones (e.g., Dong \& Blunt, 2009). 

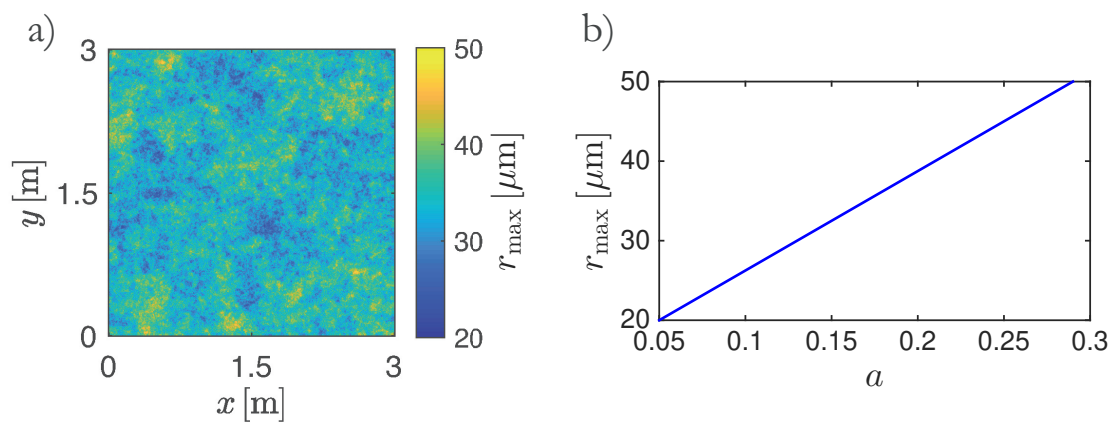

Figure 3. (a) 2D heterogeneous distribution of maximum pore radii $r_{\max }$ and (b) relationship between $r_{\max }$ and the radial factor $a$ considered in the numerical simulations.

Recall that, within each cell of the synthetic rock sample, the pore space is assumed to be composed of a fractal distribution of capillary tubes, which, in turn, are characterized by an alternation between pores with radii $r_{\min } \leq r \leq r_{\max }$ and throats with radii $r_{t h}=r a$ (Figure 1 ). The former account for most of the porosity while the latter control the flow properties. Doyen (1988) analyzed the pore space characteristics of a set of Fontainebleau sandstone samples with different porosities. The corresponding measurements show that the characteristic throat-to-pore size ratio, that is, the radial factor $a$, increases as the average pore-size increases. Furthermore, these measurements show a largely linear relationship between $a$ and the average pore-size. Based on this experimental evidence, we assume that $a$ and $r_{\max }$ are linearly related (Figure $3 \mathrm{~b}$ ). The characteristics of this relation will be further discussed in Section 3.4.

We consider a fractal dimension $D=1.465$ in agreement with the typical values for sandy porous media found by Tyler and Wheatcraft (1990). For the sake of simplicity, we assume this value to be spatially constant. The parameter $R$ is taken to be the cell side length of the computational mesh. Finally, the parameter $c$ is assumed to be spatially constant and is adjusted to obtain porosity and permeability fields whose mean values are consistent with measurements performed by Bourbié and Zinszner (1985) on Fontainebleau sandstones. The parameters employed to generate the numerical rock sample are summarized in Table 1.

Once the parameters of the hysteretic model are defined at each cell, equations (2) and (3) allow to obtain the local porosity and permeability values. As shown in Figure 4, the considered rock sample is characterized by heterogeneous porosity (Figure 4a) and 
Table 1. Mean values for the parameters of the pore-scale model employed to generate the synthetic rock sample.

\begin{tabular}{ccccc}
\hline$\left\langle r_{\max }\right\rangle$ & $\langle a\rangle$ & $c$ & $D$ & $R$ \\
\hline $33[\mu \mathrm{m}]$ & 0.16 & 0.6 & 1.465 & $5[\mathrm{~mm}]$ \\
\hline
\end{tabular}
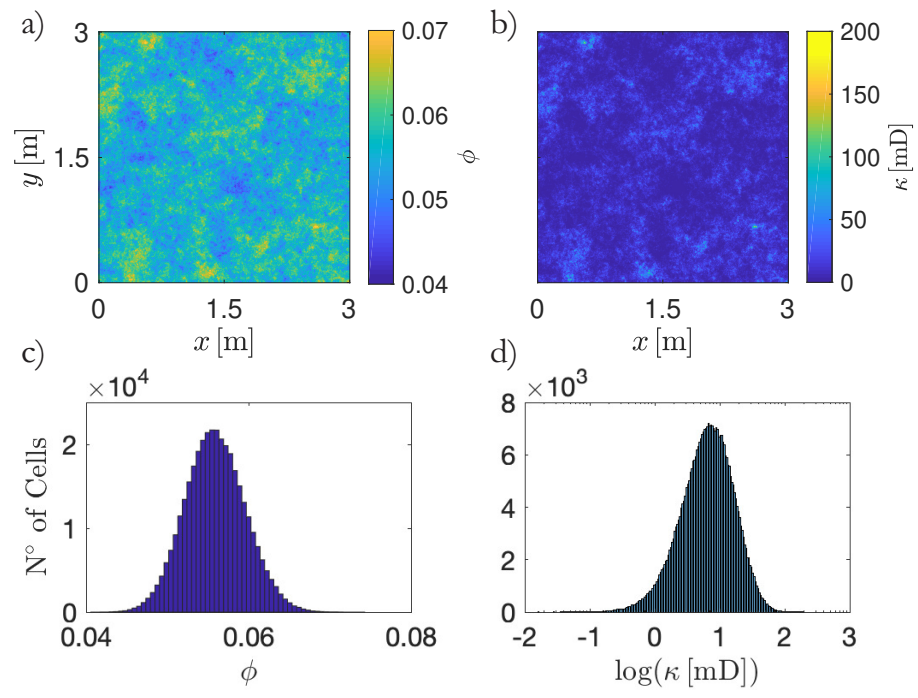

Figure 4. 2D heterogeneous a) porosity and b) permeability fields obtained from the constitutive pore-scale model. Panels c) and d) show the histograms of the corresponding fields.

permeability (Figure 4b) fields, whose mean values are $\langle\phi\rangle=5.5 \%$ and $\langle\kappa\rangle=9.35 \mathrm{mD}$, respectively. Figures $4 \mathrm{c}$ and $4 \mathrm{~d}$ show the corresponding histograms.

The pore fluids employed in the simulations are air and water, whose properties are given in Table 2. As both fluids are immiscible, their interfaces within the capillary tubes are characterized by a given contact angle $\beta$ and interfacial tension $\gamma$. The contact angle is taken as $\beta=0^{\circ}$ and the interfacial tension as $\gamma=72 \mathrm{mN} / \mathrm{m}$, in agreement with the approximate properties of water-air interfaces at a temperature of $20^{\circ} \mathrm{C}$ and at atmospheric pressure (e.g., Vargaftik et al., 1983). For the sake of simplicity, we assume that these parameters remain constant during drainage and imbibition cycles. Note that these parameters may experience small changes, whose effects are, however, beyond the scope of this work. 
Table 2. Material properties for the fluids and the solid matrix of the synthetic sandstone sample considered in this study. Adopted from Rubino and Holliger (2012), Rubino et al. (2011) and Tisato and Quintal (2013)

\begin{tabular}{lccc}
\hline \multicolumn{3}{c}{ Solid phase } \\
\hline Quartz & $K_{s}=37 \mathrm{GPa}$ & $\mu_{s}=44 \mathrm{GPa}$ & $\rho_{s}=2.64 \mathrm{~g} / \mathrm{cm}^{3}$ \\
\hline \multicolumn{3}{c}{ Fluid phases } \\
\hline Water & $K_{w}=2.3 \mathrm{GPa}$ & $\eta_{w}=0.001 \mathrm{Pas}$ & $\rho_{w}=1.0 \mathrm{~g} / \mathrm{cm}^{3}$ \\
Air & $K_{n}=1 \times 10^{-4} \mathrm{GPa}$ & $\eta_{n}=2 \times 10^{-5} \mathrm{Pas}$ & $\rho_{n}=0.001 \mathrm{~g} / \mathrm{cm}^{3}$ \\
\hline
\end{tabular}

The residual saturation $S_{w r}$ at each computational cell of the rock sample is computed following Timur's empirical equation (e.g., Timur, 1968; Mavko et al., 2009)

$$
S_{w, r}=\sqrt{\frac{8.58 \phi^{4.4}}{\kappa}}
$$

with the permeability $\kappa$ in units of Darcy [D].

Finally, the bulk and shear moduli of the dry matrix are computed at each cell using Pride's model (Pride, 2005)

$$
\begin{gathered}
K_{m}=K_{s} \frac{(1-\phi)}{\left(1+c_{s} \phi\right)}, \\
\mu_{m}=\mu_{s} \frac{(1-\phi)}{\left(1+1.5 c_{s} \phi\right)},
\end{gathered}
$$

where $K_{s}$ and $\mu_{s}$ denote the bulk and shear moduli of the solid grains, respectively. The values for these parameters are given in Table 2. The degree of cohesion between the grains is given by the so-called consolidation parameter $c_{s}$, which ranges from 2 to 20 (Pride, 2005). We use a value of $c_{s}=13$, which, according to equations (14) and (15), is consistent with the dry frame properties of low-porosity Fontainebleau sandstones (Subramaniyan et al., 2015).

Note that each cell of the numerical rock sample is characterized by a particular pair of drainage and imbibition capillary pressure-saturation curves. Hence, by assuming a constant capillary pressure state for the whole sample, one can obtain the saturation at each cell using equations (4), (5), and (6). 

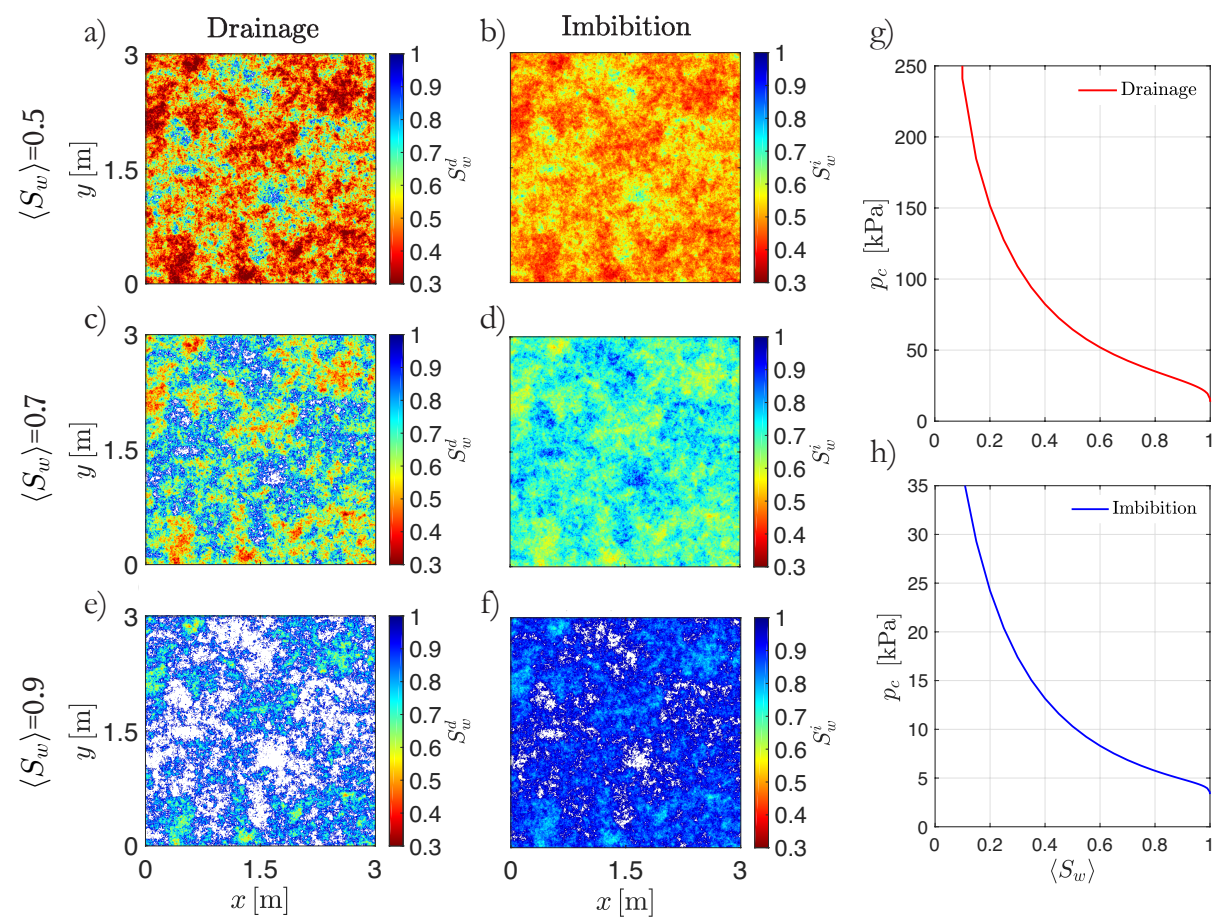

Figure 5. Saturation fields obtained through the proposed model following a drainage (left column) or an imbibition (central column) cycle for the following overall saturations levels: (a) and (b) $\left\langle S_{w}\right\rangle=0.5$; (c) and (d) $\left\langle S_{w}\right\rangle=0.7$; and (e) and (f) $\left\langle S_{w}\right\rangle=0.9$. White regions represent the zones where $K_{f} \geq 0.5 K_{w}$. Panels $(\mathrm{g})$ and (h) illustrate the capillary pressure-saturation relationships for drainage and imbibition, respectively.

\subsection{Hysteretic Saturation Patterns}

Figure 5 shows hysteretic saturation fields associated with drainage and imbibition cycles following the procedure described above. We illustrate these fields at different overall saturation values, which respond to

$$
\left\langle S_{w}\right\rangle=\frac{\sum_{i j} S_{w}\left(\Omega_{i j}\right) \phi\left(\Omega_{i j}\right)}{\sum_{i j} \phi\left(\Omega_{i j}\right)},
$$

where $\Omega_{i j}$ denotes the $i j$ th cell of the employed square computational mesh. The left column of Figure 5 illustrates the evolution of the saturation fields associated with the drainage cycle and the central column illustrates the corresponding evolution associated with the imbibition cycle. The right column shows the capillary pressure-saturation relationships for drainage (red line) and imbibition (blue line) associated with the probed sample.

Figures $5 \mathrm{a}, 5 \mathrm{c}$, and $5 \mathrm{e}$ show that during a drainage experiment the saturation field tends to present regions mainly saturated by water surrounded by zones partially sat- 
urated with air and water. To allow for a better interpretation of these fields, the regions where water saturation is $S_{w}>0.9999$ are colored with white. These regions, from now on, will be referred to as water patches and correspond to the zones where the bulk modulus of the effective pore fluid fulfills $K_{f} \geq 1 / 2 K_{w}$. That is, these are the regions that behave from a mechanical point of view as water-saturated. The remaining regions of the sample behave effectively as air-saturated. By comparison of these fields with Figure 4, we observe that the regions containing relatively high amounts of air are associated with high porosity and high permeability zones. This is expected, as the non-wetting phase percolates first into the regions where throat radii are bigger and capillary resistance is comparatively low. As a counterpart of this behavior, the wetting phase remains in the zones characterized by small throat radii. Several experimental works have observed this correlation between the non-wetting phase saturation and the zones of high porosity and permeability in heterogeneous partially saturated porous rocks (Perrin \& Benson, 2010; Shi et al., 2011; Pini et al., 2012; Alemu et al., 2013; Zhang et al., 2015)

During an imbibition process (Figures 5b, 5d, and 5f), we observe fluid distributions which are different from those obtained during drainage, thus evidencing the effects of saturation hysteresis. By performing a row-by-row comparison between the modelled imbibition and drainage saturation fields, we note that the water patches tend to appear at lower overall saturations during drainage than during imbibition. Also, we observe that during imbibition water patches have a smaller characteristic size than those associated with drainage for the same overall saturation. More importantly, the transitions between the water patches and their surroundings during imbibition are broad, partially saturated regions with smoothly varying values. However, during drainage, the spatial variation of local saturation between water patches and their surroundings is more abrupt. This characteristic of saturation hysteresis has also been observed through CT scans in the laboratory when comparing the saturation fields resulting from drainage and imbibition processes (e.g., Cadoret et al., 1998).

Figures $5 \mathrm{~g}$ and $5 \mathrm{~h}$ show the capillary pressure-overall saturation relationships for the probed sample during drainage and imbibition, respectively. We observe that the capillary pressure values during drainage are higher than those arising during imbibition for the same overall saturation value, thus exhibiting hysteresis effects. The differences in the spatial pore fluid distributions between drainage and imbibition processes are expected 
to affect the seismic attenuation and velocity dispersion characteristics due to WIFF at the mesoscopic scale.

\subsection{Seismic Attenuation and Phase Velocity Dispersion}

The effects of saturation hysteresis on seismic signatures are explored by subjecting the synthetic rock sample, saturated by the previously generated hysteretic fields, to the numerical oscillatory relaxation experiment described in Section 2.2. As a result, we obtain the frequency dependent $\mathrm{P}$-wave attenuation and phase velocity at different stages of saturation representative of drainage and imbibition experiments. It is important to remark here that as porosity and permeability fields vary smoothly in space they do not generate WIFF per se at a state of full saturation. Thus, the seismic attenuation and velocity dispersion curves analyzed in the following arise due to the presence of heterogeneities in the distribution of the pore fluids.

Arguably, one of the most studied characteristics of seismic attenuation and phase velocity dispersion is their dependence on the overall saturation (e.g., Gassmann, 1951; Lebedev et al., 2009; Monsen \& Johnstad, 2005). Figure 6 shows the phase velocity and the inverse quality factor as a function of overall saturation for drainage (red lines) and imbibition (blue lines) cycles. The seismic response is illustrated considering two frequencies: $30 \mathrm{~Hz}$ (solid lines) and $2 \mathrm{kHz}$ (dashed lines). These frequencies lie within the seismic and sonic frequency bands, respectively, which are commonly employed in field and laboratory experiments (e.g., Tisato \& Quintal, 2013; Chapman et al., 2016; Cadoret et al., 1995; Bourbié \& Zinszner, 1985; Yin et al., 1992). To allow for a better interpretation of the velocity curves, we plot in Figure 6a the Gassmann-Wood (GW) and GassmannHill (GH) models, that is, the lower and upper limits of the phase velocity, respectively (e.g., Mavko et al., 2009). These two models permit a direct evaluation of the level of dispersion associated with each curve. It is important to recall that the GW and GH models are defined for homogeneous media. As the probed sample is heterogeneous, we have employed equivalent effective properties for $K_{m}, \mu_{m}$, and $\rho_{b}$ to approximate the behavior of these curves. Further details regarding the calculation of the GW and GH curves considering equivalent effective properties are given in Appendix B.

In Figure 6a we observe that, for relatively low overall water saturations, $V_{p}$ values drop slightly or are fairly stable as the overall saturation of the sample increases. In 
this context, the average bulk density of the sample increases and its effect is comparable or greater than that of the plane wave modulus (see equation (8)). However, when the porous medium approaches full water saturation, the plane wave modulus increases drastically, thus dominating the behavior of the phase velocity. It is important to notice that, for a given overall saturation state, velocities increase with frequency due to WIFF effects. We also observe that phase velocities during drainage depart from the GW limit at lower overall saturation values than those associated with imbibition. For example, considering a relative measurement accuracy of $1 \%$ for the phase velocity (Bourbié \& Zinszner, 1985), the dispersion values expressed in Figure 6a for a frequency of $2 \mathrm{kHz}$ are experimentally measurable for saturations above 0.86 for drainage and above 0.93 for imbibition We also observe that the phase velocity values are higher during drainage than during imbibition irrespective of the frequency. A similar behavior has been observed experimentally in partially saturated rock samples by Knight and Nolen-Hoeksema (1990) and Cadoret et al. (1995). In this sense, our results show that saturation hysteresis due to the "capillary barrier" effect constitutes a physical explanation for the characteristics of the phase velocity-saturation relation during drainage and imbibition observed in these works.

Figure $6 \mathrm{~b}$ illustrates the inverse quality factor as a function of saturation for the same frequencies, that is, $30 \mathrm{~Hz}$ (solid lines) and $2 \mathrm{kHz}$ (dashed lines). We observe that the drainage process is associated with greater levels of attenuation than the imbibition cycle for most saturation levels. We also note that the attenuation values experience strong changes with frequency. Interestingly, the attenuation peaks associated with the imbibition process are located at higher overall water saturation values than the corresponding peaks during drainage. In particular, for a frequency of $30 \mathrm{~Hz}$, the drainage curve presents a peak at $\left\langle S_{w}\right\rangle=0.992$, while the imbibition curve presents a peak at $\left\langle S_{w}\right\rangle=$ 0.996. For a frequency of $2 \mathrm{kHz}$, the attenuation peaks are located at $\left\langle S_{w}\right\rangle=0.96$ for drainage and at $\left\langle S_{w}\right\rangle=0.985$ for imbibition. In this last case, the imbibition process generates greater attenuation levels than the drainage process for saturation values above 0.98. This particular characteristic of hysteresis effects on seismic signatures, where an imbibition process generates higher attenuation than a drainage process for sufficiently high overall saturations, has also been observed experimentally by Yin et al. (1992) for a partially saturated Berea sandstone. Even though there is no consensus on the lowest measurable attenuation levels in laboratory experiments, attenuation values can be 

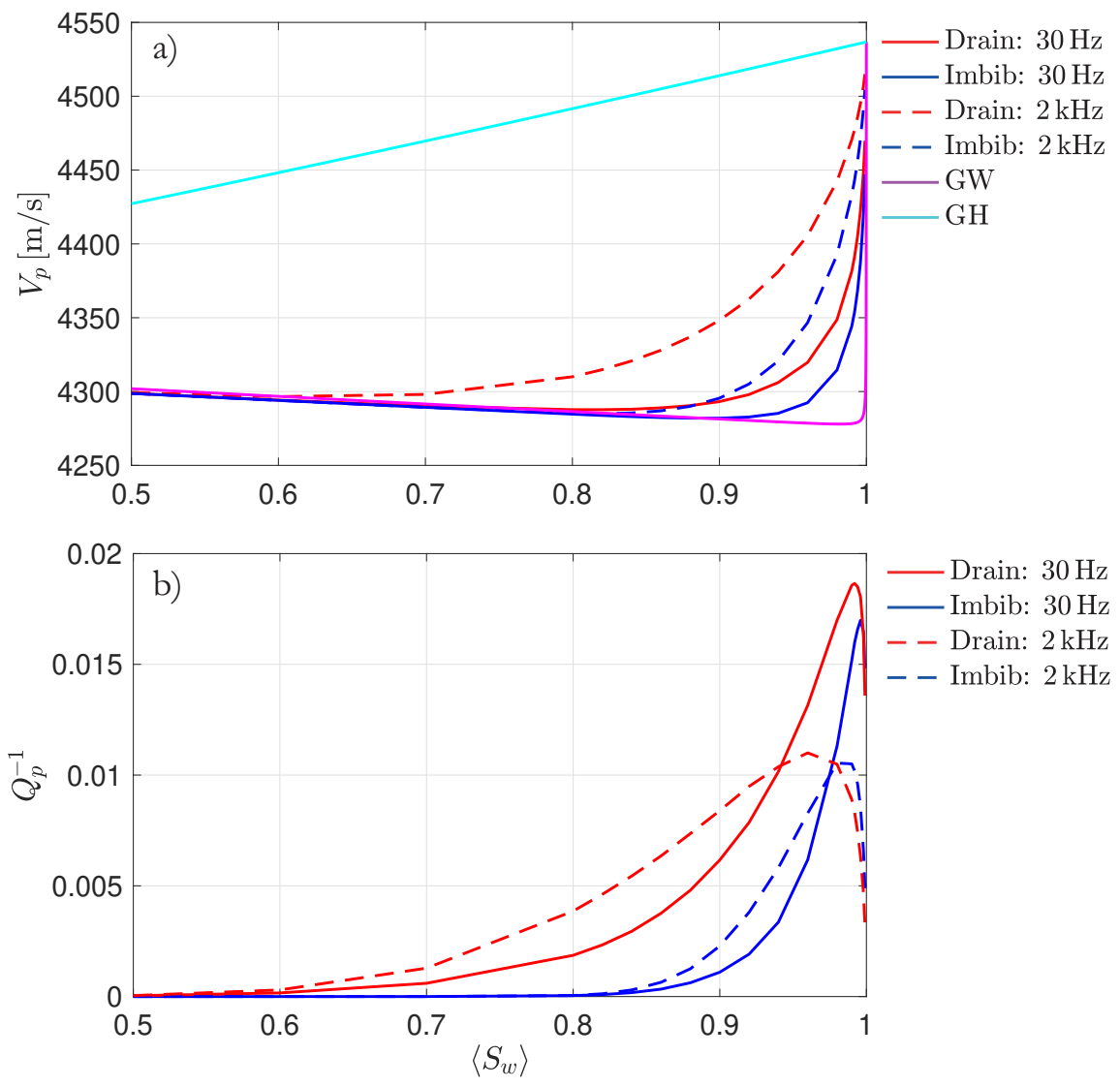

Figure 6. (a) Phase velocity and (b) inverse quality factor for imbibition (blue lines) and drainage (red lines) processes as functions of overall saturation. We consider two different frequencies: $30 \mathrm{~Hz}$ (solid lines) and $2 \mathrm{kHz}$ (dashed lines). For comparison, we also show (a) the Gassmann-Wood (GW) and Gassmann-Hill (GH) models (see Appendix B). 
measured experimentally for $1 / Q$-values above 0.003 (Tisato \& Madonna, 2012). Hence, the attenuation levels expressed in Figure $6 \mathrm{~b}$ are experimentally measurable for overall water saturations above 0.84 in drainage experiments and above 0.94 in imbibition experiments.

For a more complete analysis, we display in Figure 7 and 8 the inverse quality factor $Q_{p}^{-1}$ and phase velocity $V_{p}$ as functions of frequency for both drainage and imbibition cycles. Figure 7 shows the corresponding results for both drainage (red lines) and imbibition (blue lines) cycles as a function of frequency for overall saturation values of $\left\langle S_{w}\right\rangle=0.6$ (solid lines), $\left\langle S_{w}\right\rangle=0.7$ (circles), and $\left\langle S_{w}\right\rangle=0.8$ (dashed lines). In general, we observe in Figures $7 \mathrm{a}$ and $7 \mathrm{~b}$ that attenuation and dispersion values increase with saturation. The reasoning for this is twofold. On the one hand, as the overall water saturation of the sample increases, water patches occupy a larger portion of the medium. It is broadly known, even in simple analytical scenarios, such as, White's model (White, 1975), that higher overall water saturation values result in stronger WIFF effects. On the other hand, as the overall saturation of the sample increases, compressibility contrasts between the water patches and their surroundings increases. Consequently, the deformation caused by a passing seismic wavefield generates stronger pressure gradients and dissipation due to WIFF. Particularly, in Figure 7a, we note that $Q_{p}^{-1}$ values associated with the drainage cycle (red lines) present higher values than those associated with the imbibition cycle (blue lines), which show almost negligible attenuation values. Correspondingly, in Figure 7b, velocity dispersion is higher during drainage than during imbibition. Nevertheless, the heterogeneous saturation distributions for the overall saturations illustrated in Figure 7 produce relatively low levels of seismic attenuation and dispersion due to WIFF. Notably, attenuation levels are, at best, experimentally measurable only during drainage and for $\left\langle S_{w}\right\rangle=0.8$.

Figure 8 shows the seismic response for overall water saturation levels greater than 0.9. The saturation fields associated with both drainage and imbibition cycles are displayed in the right panels. Recall that the regions that behave effectively as water saturated patches, that is, the zones where $K_{f} \geq 0.5 K_{w}$, are colored in white. Figures $8 \mathrm{a}$ and $8 \mathrm{~b}$ show the attenuation and phase velocity curves as a function of frequency for an overall saturation state of $\left\langle S_{w}\right\rangle=0.9$. Again, we observe that $Q_{p}^{-1}$ and $V_{p}$ values associated with the drainage cycle (red solid lines) present higher values than those associated with the imbibition cycle (blue solid lines). Figures $8 \mathrm{c}$ and $8 \mathrm{~d}$ show that for an 

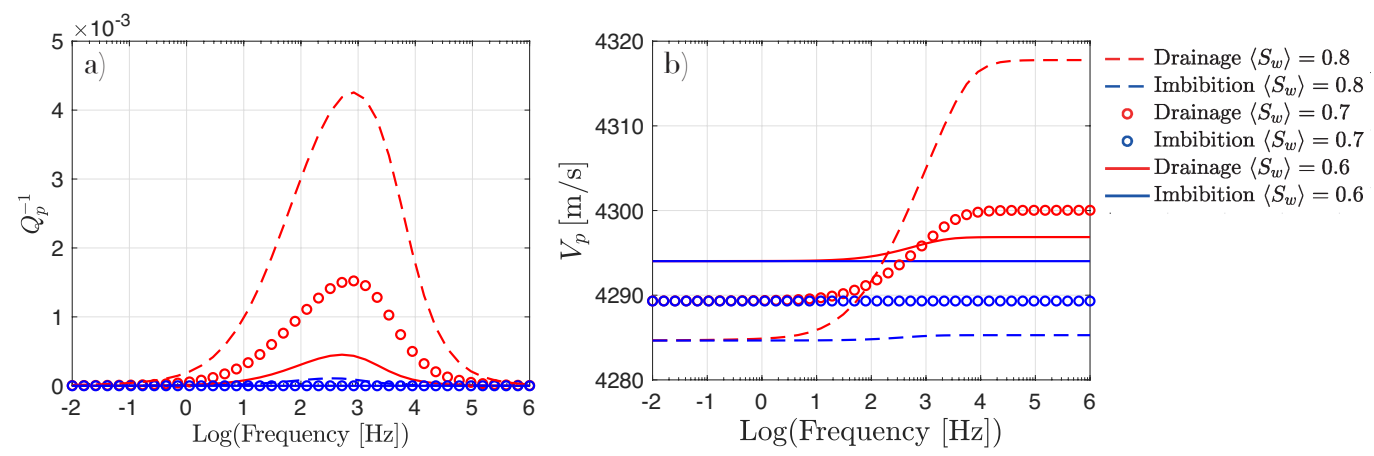

Figure 7. (a) Inverse quality factor and (b) phase velocity for imbibition (blue lines) and drainage (red lines) processes as functions of frequency. We consider three cases with different overall saturation values: $\left\langle S_{w}\right\rangle=0.6$ (solid lines), $\left\langle S_{w}\right\rangle=0.7$ (circles), and $\left\langle S_{w}\right\rangle=0.8$ (dashed lines).

overall saturation of $\left\langle S_{w}\right\rangle=0.96$, attenuation and phase velocity values are higher than for $\left\langle S_{w}\right\rangle=0.9$. However, the discrepancy between the attenuation and phase velocity curves associated with drainage and imbibition, that is, the effect of the hysteresis on the seismic signatures, is reduced. Finally, Figures $8 \mathrm{e}$ and $8 \mathrm{f}$ show the seismic behavior of the sample for an overall saturation of $\left\langle S_{w}\right\rangle=0.998$. Both attenuation and phase velocity dispersion are considerably higher than in the previous cases. We observe in Figure $8 \mathrm{e}$ that the hysteresis, that is, the difference between drainage and imbibition curves, is further reduced. Hence, Figure 8 shows that the hysteresis effect on the seismic signatures decreases as the porous medium reaches full saturation. In fact, the local imbibition and drainage capillary pressure-saturation curves approach each other in the limit of full saturation (Figure 2). Interestingly, we observe in Figure 8e that, for frequencies above $20 \mathrm{~Hz}$, the inverse quality factor associated with imbibition is higher than the one associated with drainage. This behavior was observed previously in Figure 6 for such saturation values. This analysis shows that saturation hysteresis effects on seismic signatures are highly complex and that, even if drainage processes tend to be associated with higher levels of dissipation due to WIFF, this might not be the case when the porous medium is close to the full saturation.

We have observed in Figure 8 that the frequency associated with the maximum attenuation value, $f_{c}$, exhibits different values for drainage and imbibition and, also, that these values vary with the overall saturation. This is an interesting phenomenon, the anal- 
a)
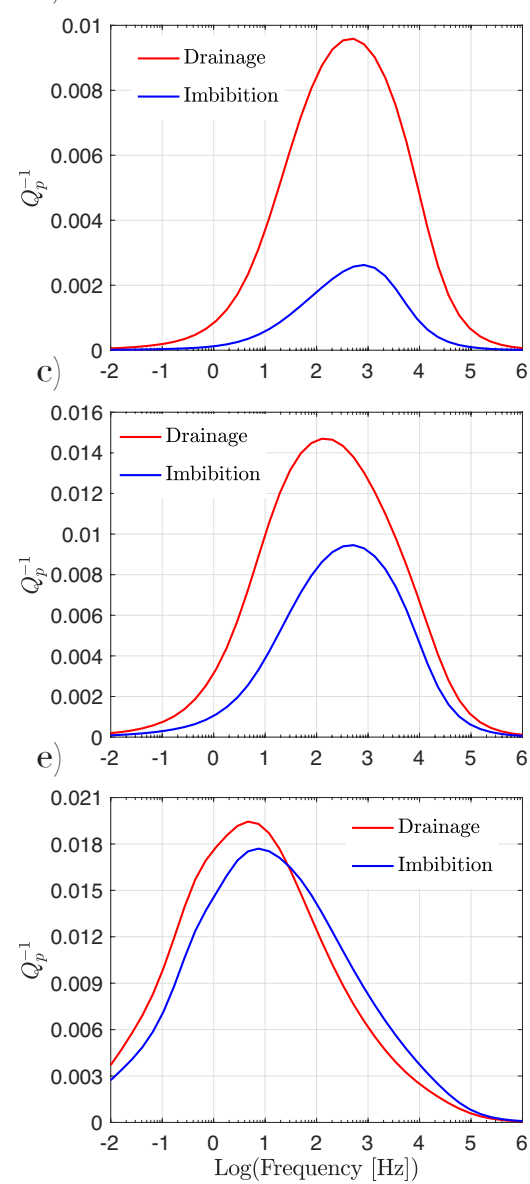

b)
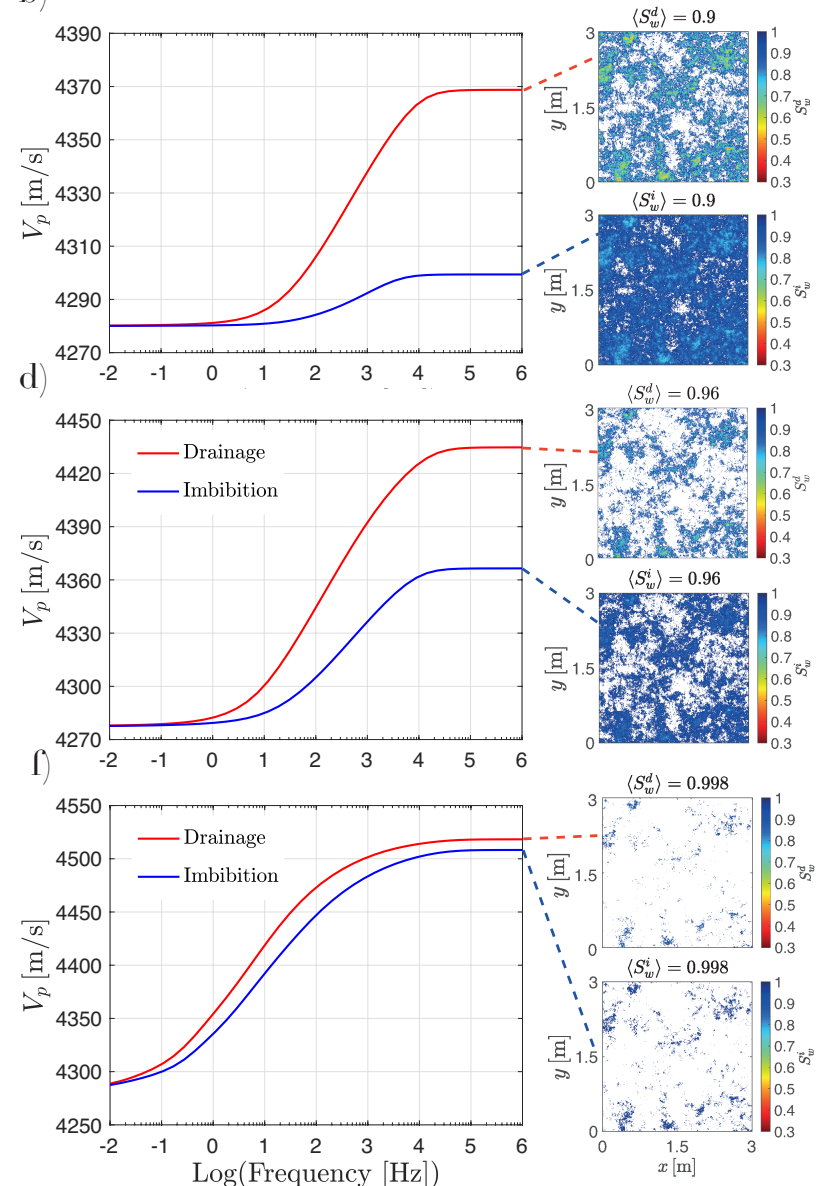

Figure 8. Inverse quality factor and phase velocity for imbibition (blue solid lines) and drainage (red solid lines) processes as functions of frequency. We consider three cases with different overall saturation values: (a) and (b) $\left\langle S_{w}\right\rangle=0.9$, (c) and (d) $\left\langle S_{w}\right\rangle=0.96$, and (e) and (f) $\left\langle S_{w}\right\rangle=0.998$. On the right-hand side, and connected to the corresponding dispersion curves, we plot the saturation fields for both drainage and imbibition processes. White regions represent the zones where $K_{f} \geq 0.5 K_{w}$. 

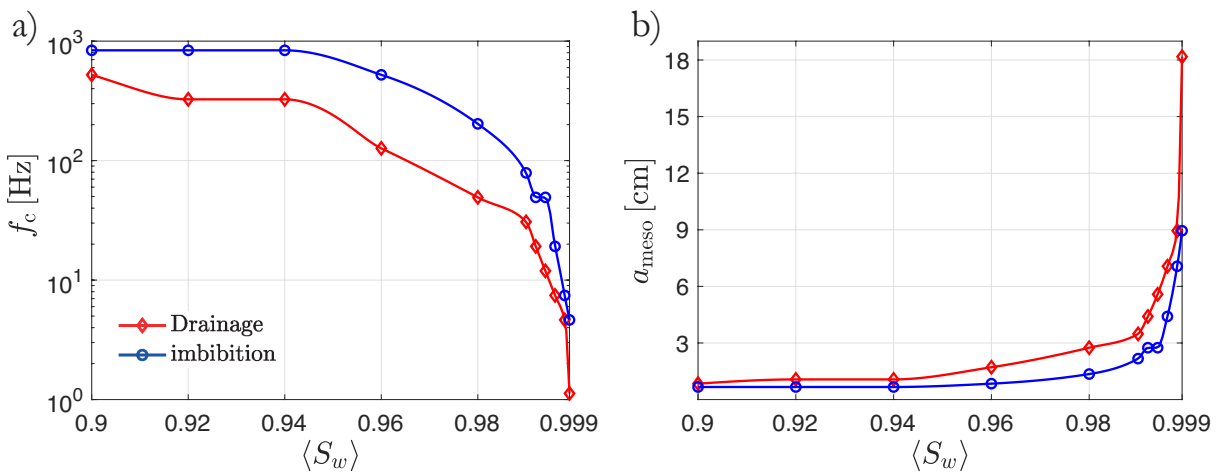

Figure 9. (a) Critical frequency $f_{\mathrm{c}}$ and (b) characteristic patch size $a_{\text {meso }}$ as a function of overall saturation $\left\langle S_{w}\right\rangle$.

ysis of which, as further explained below, permits to estimate the characteristic size of the water saturated patches. Figure 9a illustrates the variation of $f_{c}$ with the overall saturation during drainage (red line) and imbibition (blue line). The $f_{c^{-}}$-values are obtained from the previously described attenuation curves (Figure 8 ). We observe that $f_{c}$ decreases with increasing overall saturation for both drainage and imbibition cycles. Also, we observe that drainage processes are associated with lower $f_{c^{-}}$values than imbibition processes for the same overall saturation. In order to reconcile this, it is important to recall that (e.g., Müller et al., 2010)

$$
f_{c} \simeq \frac{D}{2 \pi a_{\text {meso }}^{2}}
$$

where $D$ is the diffusivity of the material composing the heterogeneities where energy dissipation occurs (equation (A8)) and $a_{\text {meso }}$ is their characteristic size. By looking at the panels on the right-hand side of Figure 8, it can be argued that the reduction of $f_{c}$ with overall saturation is caused by an increase in the characteristic size of the water patches with increasing water saturation. Also, patches during drainage seems to be larger than during imbibition (Figure 8), which explains the fact that the $f_{c}$-values are higher for the latter case.

Notably, equation (17) permits to estimate the characteristic size of the heterogeneities involved in the WIFF process by using the $f_{c}$-values (Figure $9 \mathrm{a}$ ) and an approximate value for the diffusivity $D$. The latter is obtained by considering the mean porosity of the rock in equations (14) and (15), and the fluid properties of water when computing equation (A8). In this context, equation (17) constitutes an approximation and, as noted by Carcione 
et al. (2003) in the context of White's spherical patch model (White, 1975), a more representative estimate of the characteristic water patch size is $\sim 2 a_{\text {meso }}$, which is the distance between air patches. Figure $9 \mathrm{~b}$ shows the behavior of $a_{\text {meso }}$ during drainage (red line) and imbibition (blue line) cycles. An important feature of Figure $9 \mathrm{~b}$ is that that the values of the characteristic patch size $2 a_{\text {meso }}$ during drainage are larger than those associated with imbibition processes. For overall saturations varying from 0.9 to 0.999 the characteristic patch size $2 a_{\text {meso }}$ increases from $1.7 \mathrm{~cm}$ to $36 \mathrm{~cm}$ for drainage and from $1.3 \mathrm{~cm}$ to $18 \mathrm{~cm}$ for imbibition. However, by qualitatively comparing these values with the water patches illustrated in the panels on the right-hand side of Figure 8, we note that the latter are larger than the former. This discrepancy is expected, as in presence of highly irregular patches, such as the ones modelled in this work, fluid pressure diffusion takes place at different scales and, thus, several patch sizes can be defined. In this sense, the $a_{\text {meso-values derived from equation (17) are representative of the spatial scales }}$ involved in the diffusion process for the frequency $f_{c}$.

\subsection{Effects of Throat-to-Pore Size Ratio on WIFF}

The radial factor $a$, that is, the throat-to-pore size ratio, constitutes a key porescale parameter when exploring the hysteretic behavior of a porous medium. Local variations of the radial factor have an impact on the permeability and, as pore throats act as "capillary barriers" to the flow of the non-wetting phase, on the characteristics of the saturation field during drainage processes. The pressure relaxation process induced by a passing $\mathrm{P}$-wave is highly sensitive to changes in these properties and, hence, the effects of the throat-to-pore size ratio on the resulting seismic signatures should be further analyzed.

We shall explore the effects of the radial factor on WIFF considering a simple numerical experiment. That is, we propose to increase all local values of the radial factor $a\left(\Omega_{i j}\right)$ by a fixed amount maintaining the original standard deviation and, thus, maintaining the degree of spatial heterogeneity. Hence, we make the throat-to-pore size ratio bigger throughout the medium. As further explained below, this experiment is performed by changing the relationship between $a$ and $r_{\max }$ displayed in Figure $3 \mathrm{~b}$.

In Figure 10a, the solid line represents the relationship between $a$ and $r_{\max }$ considered in the previous sections, where $a$ is characterized by a standard deviation of $\sigma(a)=$ 
a)

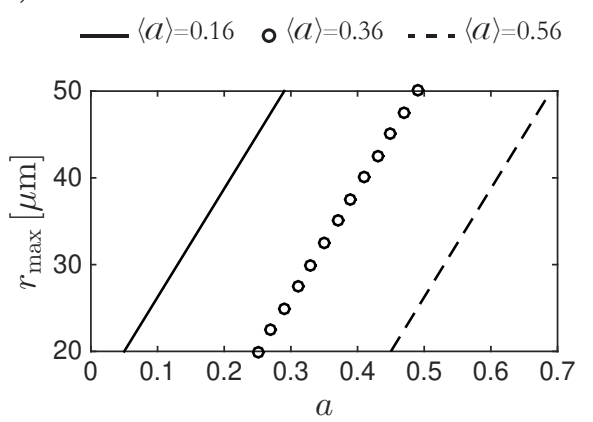

b)
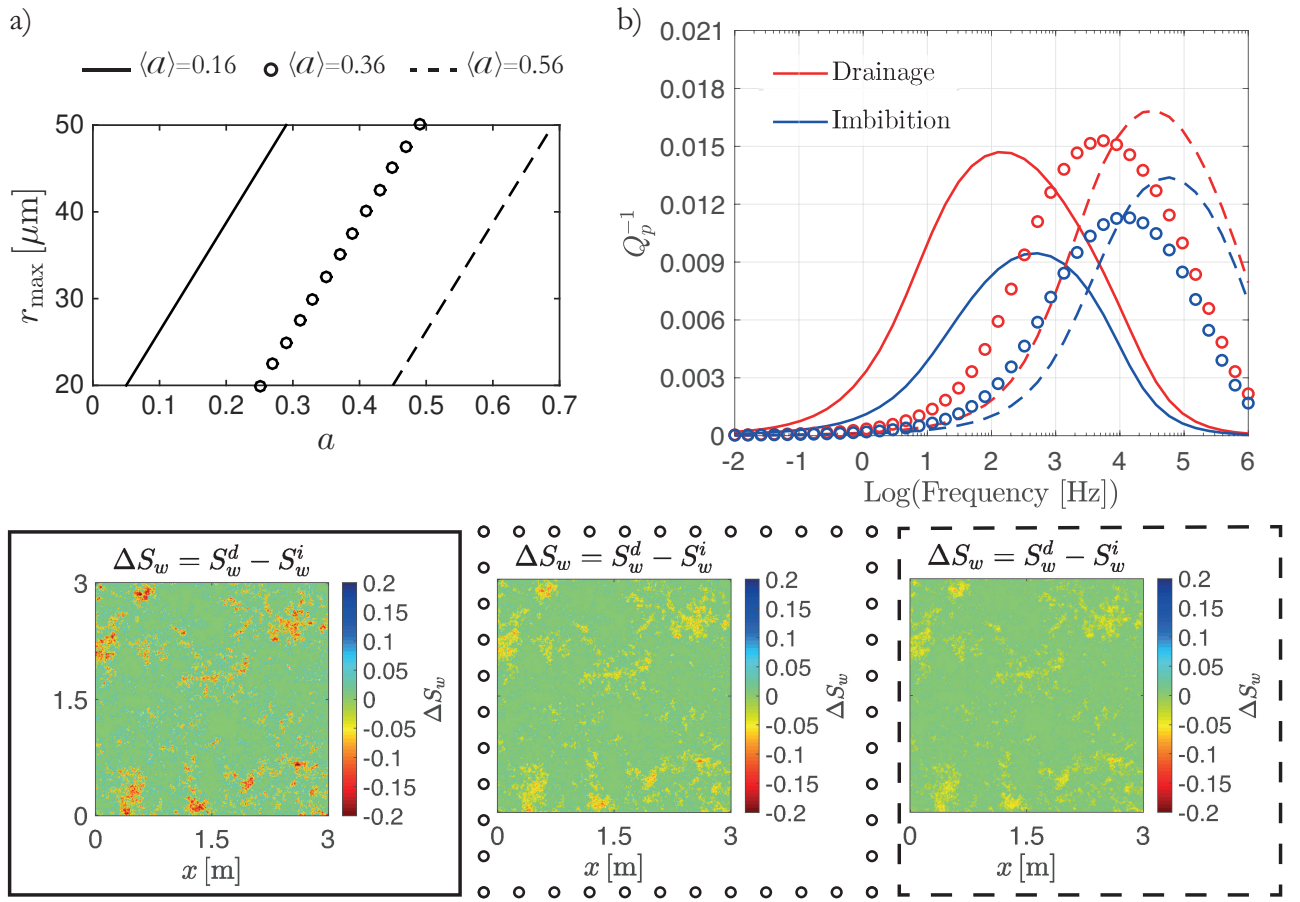

Figure 10. (a) Relationships between $r_{\max }$ and $a$ with the same standard deviation. The solid line results in a $a$ field with $\langle a\rangle=0.16$, while the circled and dashed lines correspond to $\langle a\rangle=0.36$ and $\langle a\rangle=0.56$, respectively. (b) Inverse quality factor for imbibition (blue lines) and drainage (red lines) processes as a function of frequency for the cases considered in panel (a). At the bottom, and framed by the corresponding features (solid lines, circles, and dashed lines), we plot the difference between drainage and imbibition saturation fields for each case. 
0.032 and a mean value of $\langle a\rangle=0.16$. The circled and dashed lines represent two new relationships characterized by the same standard deviation but with mean values of $\langle a\rangle=$ 0.36 and $\langle a\rangle=0.56$, respectively. Note that, these new relationships are nothing but an increase in the original radial factor values of 0.2 and 0.4 , respectively.

The effects of these changes on the seismic attenuation curves are illustrated in Figure $10 \mathrm{~b}$, where we use red colored lines for drainage and blue lines for imbibition. The overall saturation of the sample for this particular example is 0.96. The features employed to represent the different relationships in Figure 10a, that is, solid lines, circles, and dashed lines, are maintained in Figure 10b to represent the corresponding attenuation curves. Note that the bottom panels show the difference between drainage and imbibition saturation fields for each case using the same features (solid lines, circles, and dashed lines) on the corresponding frames. On one hand in Figure 10b, we observe that, as the mean radial factor increases, the characteristic frequency shifts to higher values. This is expected, as the permeability of the sample increases for increasing $\langle a\rangle$ values. The increment in the local permeability values affects the diffusivity (equation (A8)) and, thus, the characteristic frequency is shifted towards higher values (see equation (17)). On the other hand, the increase in the porosity impacts on the effective bulk moduli of the medium, making the rock more compliant, and, consequently, the attenuation levels rise. We also observe that the difference between the attenuation curves associated with imbibition and drainage cycles is reduced as the radial factor increases. It is important to recall that the hysteretic behavior is included in the constitutive model considering constrictive segments or throats in the pore scale geometry (see Figure 1). As the radial factor increases, the pore-scale geometry approaches that of a non-hysteretic straight-tube and, thus, hysteresis effects tend to disappear. This is also observed in the bottom panels, where the differences between drainage and imbibition water saturation fields is reduced as the radial factor increases. Correspondingly, the immediate effect of increasing the radial factor is a reduction of the hysteresis effect on the saturation fields and on the seismic signatures. Several phenomena, such as clogging, and precipitation/dissolution of minerals within the pore space have the potential to fundamentally change the characteristic pore-to-throat size ratio of a porous formation. Our numerical experiments suggest that seismic attenuation and velocity dispersion due to mesoscopic WIFF in partially saturated media is likely to be sensible to the effects of these processes. 


\section{Conclusions}

In this work, we have implemented a numerical procedure to explore saturation hysteresis effects on seismic attenuation and phase velocity dispersion due to WIFF. To do so, we generated a heterogeneous synthetic rock sample whose hydraulical properties are computed by means of a recently proposed hysteretic constitutive model. Through this approach, we obtained a set of hysteretic saturation fields representative of drainage and imbibition cycles by assuming a set of capillary equilibrium states. Considering these hysteretic fields, we then applied a numerical upscaling procedure to quantify seismic attenuation and velocity dispersion due to WIFF.

The numerical analysis shows that the hysteresis associated with drainage and imbibition processes has a significant impact on the seismic signatures. Consequently, hysteresis effects should be considered to allow for an adequate seismic characterization of partially saturated media. We also observe that phase velocities during drainage depart from the GW limit at lower overall saturation levels than during imbibition. In general, we observe that energy dissipation due to WIFF during the drainage cycle is greater than during the imbibition cycle. An analysis of the hysteretic saturation fields allowed us to demonstrate that this feature is due to the discrepancy in the spatial characteristics of the resulting saturation fields. Drainage processes tend to generate fluid patches at lower overall saturations and with more abrupt transitions towards their partially saturated surroundings than imbibition processes. This, in turn, generates more pronounced compressibility contrasts and stronger WIFF effects. Also, we observed that drainage processes tend to generate water patches with greater characteristic size than imbibition processes. Consequently, the characteristic frequency of the attenuation curve associated with drainage processes is lower than the corresponding frequency associated with imbibition. Nevertheless, as the sample approaches the limit of full saturation, hysteresis effects on WIFF tend to decrease and the discrepancy between the seismic signatures associated with drainage and imbibition processes is reduced. In this context, imbibition processes can indeed generate more attenuation than drainage processes for sufficiently high frequencies. The characteristics of the hysteretic saturation fields and of the associated seismic signatures modelled with the proposed approach were previously observed in several laboratory experiments. Hence, saturation hysteresis due to the "capillary barrier" effect constitutes a plausible explanation for the observed behavior of seis- 
mic attenuation and the phase velocity during drainage and imbibition processes in partially saturated porous media.

Our results also illustrate the importance of the throat-to-pore size ratio or radial factor, as it greatly impacts the characteristics of the pore fluid distribution during drainage and imbibition processes. In general, larger values of the radial factor generate less constrictive throats. This, in turn, increases the porosity and the permeability and reduces the effects of the saturation hysteresis on the seismic signatures. Hence, seismic signatures in partially saturated environments during drainage and imbibition processes are sensitive to changes in the pore-scale characteristics of the rock frame.

\section{Appendix A Numerical Oscillatory Relaxation Test for Computing Seis- mic Attenuation due to WIFF}

To compute the response of the sample subjected to the considered relaxation test, we solve Biot's quasi-static poroelastic equations (Biot, 1941), which in the space-frequency domain results in the following system of equations

$$
\begin{gathered}
\nabla \cdot \boldsymbol{\tau}=0, \\
\nabla p_{f}=-i \omega \frac{\eta_{f}}{\kappa} \boldsymbol{w},
\end{gathered}
$$

where $\boldsymbol{\tau}$ represents the total stress tensor, $p_{f}$ is the pressure of the fluid, and $\boldsymbol{w}$ the relative fluid-solid displacement.

Equations (A1) and (A2) are coupled through the stress-strain constitutive relations (Biot, 1962)

$$
\begin{gathered}
\boldsymbol{\tau}=2 \mu_{m} \boldsymbol{\epsilon}+\boldsymbol{I}\left(\lambda_{c} \nabla \cdot \boldsymbol{u}-\alpha M \zeta\right), \\
p_{f}=-\alpha M \nabla \cdot \boldsymbol{u}+M \zeta,
\end{gathered}
$$

where $\boldsymbol{I}$ is the identity matrix, $\boldsymbol{u}$ the solid displacement, and $\zeta=-\nabla \cdot \boldsymbol{w}$ a measure of the local change in the fluid content. The strain tensor is given by $\boldsymbol{\epsilon}=\frac{1}{2}\left(\nabla \boldsymbol{u}+(\nabla \boldsymbol{u})^{\mathrm{T}}\right)$, with $\mathrm{T}$ denoting the transpose operator. The poroelastic Biot-Willis parameter $\alpha$, the fluid storage coefficient $M$, and the Lamé parameter $\lambda_{c}$ are given by (e.g., Rubino et al., 2009)

$$
\begin{gathered}
\alpha=1-\frac{K_{m}}{K_{s}} \\
M=\left(\frac{\alpha-\phi}{K_{s}}+\frac{\phi}{K_{f}}\right)^{-1},
\end{gathered}
$$


and

$$
\lambda_{c}=K_{m}+\alpha^{2} M-\frac{2}{3} \mu_{m},
$$

respectively. The diffusivity $D$, employed in equation (17), can be expressed in terms of the poroelastic properties of the fluid saturated porous rock (e.g., Rubino \& Holliger, 2012)

$$
D=\frac{\kappa}{\eta_{f}}\left(\frac{M H-\alpha^{2} M^{2}}{H}\right)
$$

with $H=\lambda_{c}+2 \mu_{m}$.

Equations (A1) through (A4) are numerically solved under adequate boundary conditions. Let $\Omega_{\text {sub }}$ be a square domain that represents the sample subjected to the oscillatory test. In addition, $\Gamma_{\text {sub }}$ is the boundary of $\Omega_{\text {sub }}$. We consider the following boundary conditions

$$
\begin{aligned}
\boldsymbol{u} \cdot \boldsymbol{\nu}_{\mathrm{sub}} & =-\Delta u, \quad(x, y) \in \Gamma_{\mathrm{sub}}^{T}, \\
\boldsymbol{u} \cdot \boldsymbol{\nu}_{\mathrm{sub}} & =0, \quad(x, y) \in \Gamma_{\mathrm{sub}}^{L} \cup \Gamma_{\mathrm{sub}}^{R} \cup \Gamma_{\mathrm{sub}}^{B}, \\
\left(\boldsymbol{\tau} \cdot \boldsymbol{\nu}_{\mathrm{sub}}\right)^{\mathrm{T}} \cdot \boldsymbol{\chi}_{\mathrm{sub}} & =0, \quad(x, y) \in \Gamma_{\mathrm{sub}}, \\
\boldsymbol{w} \cdot \boldsymbol{\nu}_{\mathrm{sub}} & =0, \quad(x, y) \in \Gamma_{\mathrm{sub}},
\end{aligned}
$$

where $\Gamma_{\text {sub }}^{L}, \Gamma_{\text {sub }}^{R}, \Gamma_{\text {sub }}^{B}$, and $\Gamma_{\text {sub }}^{T}$ are the left, right, bottom, and top boundaries of the sample, respectively, and $\boldsymbol{\nu}_{\text {sub }}$ and $\boldsymbol{\chi}_{\text {sub }}$ are the unit normal and the unit tangent of the sample's boundary $\Gamma_{\text {sub }}$, respectively.

A finite-element procedure is then employed to solve equations (A1)-(A4) under the above boundary conditions. We use bilinear functions to approximate the solid displacement vector and a closed sub-space of the vector part of the Raviart-Thomas-Nedelec space of zero order for representing the relative fluid displacement (Raviart \& Thomas, 1977; Nedelec, 1980). Assuming that the volume average responses of the probed sample can be represented by an equivalent homogeneous isotropic viscoelastic solid, the resulting averages over the sample's volume of the vertical components of the stress and strain fields, $\left\langle\tau_{y y}(\omega)\right\rangle$ and $\left\langle\epsilon_{y y}(\omega)\right\rangle$, allow to compute a complex-valued frequency-dependent equivalent plane-wave modulus

$$
M_{c}(\omega)=\frac{\left\langle\tau_{y y}(\omega)\right\rangle}{\left\langle\epsilon_{y y}(\omega)\right\rangle}
$$




\section{Appendix B Velocity Estimates for the Relaxed and Unrelaxed States}

The dependence of the phase velocity on the overall saturation is usually described employing Gassmann's model (Gassmann, 1951), which assumes that the porous medium is homogeneous and saturated by a single fluid phase. If multiple fluid phases are present in the pore space, the effective fluid bulk modulus can be estimated using Wood's and Hill's formulae (Mavko et al., 2009). These expressions allow to obtain the relaxed and unrelaxed state limits for the phase velocity, respectively. Correspondingly, if the frequency is sufficiently low such that the fluid pressure is equilibrated during a wave cycle, equation (11) can be applied to calculate an effective fluid bulk modulus of the medium $K_{f}^{\mathrm{GW}}$. Then, the effective plane wave modulus of the rock can be obtained from the GassmannWood relation

$$
H^{\mathrm{GW}}=K_{m}+\frac{4}{3} \mu_{m}+\alpha^{2} M\left(K_{f}^{\mathrm{GW}}\right),
$$

where $M\left(K_{f}^{\mathrm{GW}}\right)$ implies that the fluid storage coefficient is computed using the properties of the effective fluid. Conversely, the effective plane wave modulus in the high-frequency limit is given by Hill's average (e.g., Johnson, 2001)

$$
H^{\mathrm{GH}}=\frac{\left\langle S_{w}\right\rangle}{H^{w}}+\frac{\left(1-\left\langle S_{w}\right\rangle\right)}{H^{n}},
$$

where $H^{q}=K_{m}+\frac{4}{3} \mu_{m}+\alpha^{2} M\left(K_{q}\right)$, with $q=w, n$. Consequently, the GassmannWood and Gasmann-Hill relaxed and unrelaxed phase velocity limits correspond to

$$
V_{p}^{\mathrm{GW}}=\sqrt{\frac{H^{\mathrm{GW}}}{\rho_{b}}}, \quad \text { and } \quad V_{p}^{\mathrm{GH}}=\sqrt{\frac{H^{\mathrm{GH}}}{\rho_{b}}},
$$

respectively. Due to the fact that the sample considered in this work is not homogeneous, effective equivalent properties for these two models are required. We then compute $K_{m}^{\mathrm{eq}}$, $\rho_{b}^{\mathrm{eq}}, \mu_{m}^{\mathrm{eq}}, \alpha^{\mathrm{eq}}$, and $M^{\mathrm{eq}}$ employing the mean porosity $\langle\phi\rangle$ in the relations (14) and (15). These values are then employed in the equations (B1) and (B2).

\section{Acknowledgments}

Part of this work was completed within the Swiss Competence Center for Energy ResearchSupply of Electricity with support of Innosuisse. J. G. R. gratefully acknowledges the financial support received from the Agencia Nacional de Promoción Científica y Tecnológica of Argentina (PICT 2017-2976). The authors thank Eva Caspari for enlightening discussions and two anonymous reviewers for lucid comments and insightful suggestions. The hysteretic saturation fields and the seismic attenuation and velocity dispersion data for this paper are available from https://github.com/PoroelasticityUNIL/Hysteresis. 


\section{References}

Akbar, N., Mavko, G., Nur, A., \& Dvorkin, J. (1994). Seismic signatures of reservoir transport properties and pore fluid distribution. Geophysics, 59(8), 12221236.

Alemu, B. L., Aker, E., Soldal, M., Johnsen, Ø., \& Aagaard, P. $\quad$ (2013). $\quad$ Effect of sub-core scale heterogeneities on acoustic and electrical properties of a reservoir rock: $\mathrm{A} \mathrm{CO}_{2}$ flooding experiment of brine saturated sandstone in a computed tomography scanner. Geophys. Prospect., 61, 235-250.

Ba, J., Carcione, J. M., \& Sun, W. (2015). Seismic attenuation due to heterogeneities of rock fabric and fluid distribution. Geophys. J. Int., 202(3), 18431847.

Bear, J. (1972). Dynamics of Fluids in Porous Media. New York: Am. Elsevier.

Biot, M. A. (1941). General theory of three-dimensional consolidation. J. Appl. Phys., 12, 155-164. doi: 10.1063/1.1712886

Biot, M. A. (1962). Mechanics of deformation and acoustic propagation in porous media. J. Appl. Phys., 33(4), 1482-1498. doi: 10.1063/1.1728759

Borcherdt, R. D. (2009). Viscoelastic Waves in Layered Media. Cambridge University Press.

Bourbié, T., \& Zinszner, B. (1985). Hydraulic and acoustic properties as a function of porosity in Fontainebleau sandstone. J. Geophys. Res., 90, 11524-11532.

Brooks, R., \& Corey, A. (1964). Hydraulic Properties of Porous Media. Colorado State University.

Cadoret, T., Marion, D., \& Zinszner, B. (1995). Influence of frequency and fluid distribution on elastic wave velocities in partially saturated limestones. J. Geophys. Res., 100(B6), 9789-9803.

Cadoret, T., Mavko, G., \& Zinszner, B. (1998). Fluid distribution effect on sonic attenuation in partially saturated limestones. Geophysics, 63, 154-160.

Carcione, J. M., Helle, H. B., \& Pham, N. H. (2003). White's model for wave propagation in partially saturated rocks: Comparison with poroelastic numerical experiments. Geophysics, 68(4), 1389-1398.

Chapman, S., Tisato, N., Quintal, B., \& Holliger, K. ～(2016). Seismic attenuation in partially saturated Berea sandstone submitted to a range of confining pressures. J. Geophys. Res., 121(3), 1664-1676. 
Dong, H., \& Blunt, M. J. (2009). Pore-network extraction from micro-computerizedtomography images. Phys. Rev. E, 80(3), 036307.

Doyen, P. M. (1988). Permeability, conductivity, and pore geometry of sandstone. J. Geophys. Res. Solid Earth, 93(B7), 7729-7740.

Gassmann, F. (1951). Über die Elastizität poröser Medien. Vierteljahresschr. Naturforsch. Ges. Zürich, 96, 1-23.

Guarracino, L. (2007). Estimation of saturated hydraulic conductivity $k_{s}$ from the van genuchten shape parameter $\alpha$. Water Resour. Res., 43(11).

Guarracino, L., Rötting, T., \& Carrera, J. (2014). A fractal model to describe the evolution of multiphase flow properties during mineral dissolution. Adv. Water Resour., 67, 78-86.

Hogarth, W. L., Hopmans, J., Parlange, J. Y., \& Haverkamp, R. (1988). Application of a simple soil-water hysteresis model. J. Hydrol., 98(1-2), 21-29.

Johnson, D. L. (2001). Theory of frequency dependent acoustics in patchy-saturated porous media. J. Acoust. Soc. Am., 110, 682-694. doi: 10.1121/1.1381021

Juanes, R., Spiteri, E. J., Orr, F. M., \& Blunt, M. J. (2006). Impact of relative permeability hysteresis on geological $\mathrm{CO}_{2}$ storage. Water Resour. Res., 42(12).

Kearey, P., Brooks, M., \& Hill, I. (2013). An Introduction to Geophysical Exploration. John Wiley \& Sons.

Knight, R., \& Nolen-Hoeksema, R. (1990). A laboratory study of the dependence of elastic wave velocities on pore scale fluid distribution. Geophys. Res. Lett., $17(10), 1529-1532$.

Krause, M., Krevor, S., \& Benson, S. M. (2013). A procedure for the accurate determination of sub-core scale permeability distributions with error quantification. Transport Porous Med., 98(3), 565-588.

Krevor, S., Pini, R., Li, B., \& Benson, S. M. (2011). Capillary heterogeneity trapping of $\mathrm{CO}_{2}$ in a sandstone rock at reservoir conditions. Geophys Res. Lett., 38(15).

Lebedev, M., Toms-Stewart, J., Clennell, B., Peruvukhina, M., Shulakova, V., Paterson, L., ... Wenzlau, F. (2009). Direct laboratory observation of patchy saturation and its effects on ultrasonic velocities. The Leading Edge, 28(1), $24-27$.

Lenhard, R. J., Parker, J. C., \& Kaluarachchi, J. J. (1991). Comparing simulated 
and experimental hysteretic two-phase transient fluid flow phenomena. Water Resour. Res., 27(8), 2113-2124.

Lenormand, R. (1990). Liquids in porous media. J. Phys. Condens. Matter, 2, 7988.

Lenormand, R., Zarcone, C., \& Sarr, A. (1983). Mechanisms of the displacement of one fluid by another in a network of capillary ducts. J. Fluid Mech., 135, 337353.

Le Ravalec, M., Guéguen, Y., \& Chelidze, T. (1996). Elastic wave velocities in partially saturated rocks: Saturation hysteresis. J. Geophys. Res., 101, 837-844.

Li, B., \& Benson, S. M. (2015). Influence of small-scale heterogeneity on upward $\mathrm{CO}_{2}$ plume migration in storage aquifers. Adv. Water Resour., 83, 389-404.

Masson, Y. J., \& Pride, S. R. (2011). Seismic attenuation due to patchy saturation. J. Geophys. Res., 116, B0326. doi: 10.1029/2010JB007983

Mavko, G., Mukerji, T., \& Dvorkin, J. (2009). The Rock Physics Handbook: Tools for Seismic Analysis of Porous Media. Cambridge University Press.

Monsen, K., \& Johnstad, S. E. (2005). Improved understanding of velocitysaturation relationships using 4D computer-tomography acoustic measurements. Geophys. Prospect., 53(2), 173-181.

Müller, T. M., Gurevich, B., \& Lebedev, M. (2010). Seismic wave attenuation and dispersion resulting from wave-induced flow in porous rocks - a review. Geophysics, 75, 147-163. doi: 10.1190/1.3463417

Nakagawa, S., Kneafsey, T. J., Daley, T. M., Freifeld, B. M., \& Rees, E. V. (2013). Laboratory seismic monitoring of supercritical $\mathrm{CO}_{2}$ flooding in sandstone cores using the split hopkinson resonant bar technique with concurrent X-ray computed tomography imaging. Geophys. Prospect., 61(2), 254-269.

Nedelec, J. C. (1980). Mixed finite elements in $\mathrm{r}^{3}$. Numer. Math., 35, 315-341. doi: 10.1007/BF01396415

Papageorgiou, G., \& Chapman, M. (2015). Multifluid squirt flow and hysteresis effects on the bulk modulus-water saturation relationship. Geophys. J. Int., $203(2), 814-817$.

Perrin, J.-C., \& Benson, S. (2010). An experimental study on the influence of subcore scale heterogeneities on $\mathrm{CO}_{2}$ distribution in reservoir rocks. Transport Porous Med., 82(1), 93-109. 
Pini, R., Krevor, S. C., \& Benson, S. M. (2012). Capillary pressure and heterogeneity for the $\mathrm{CO}_{2}$ /water system in sandstone rocks at reservoir conditions. $A d v$. Water Resour., 38, 48-59.

Pride, S. R. (2005). Relationships between seismic and hydrological properties. In Y. Rubin \& S. Hubbard (Eds.), Hydrogeophysics (pp. 253-290). Springer.

Raviart, P., \& Thomas, J. (1977). Mixed finite element method for 2nd order elliptic problems. Lect. Notes Math., 606, 292-315. doi: 10.1007/BFb0064470

Rubino, J. G., \& Holliger, K. (2012). Seismic attenuation and velocity despersion in heterogeneous partially saturated porous rocks. Geophys. J. Int., 188, 10881102. doi: 10.1111/j.1365-246X.2011.05291.x

Rubino, J. G., \& Holliger, K. ～(2013). $\quad$ Research note: Seismic attenuation due to wave-induced fluid flow at microscopic and mesoscopic scales. Geophys. Prospect., 882-889. doi: 10.1111/1365-2478.12009

Rubino, J. G., Ravazzoli, C. L., \& Santos, J. E. (2009). Equivalent viscoelastic solids for heterogeneous fluid-saturated porous rocks. Geophysics, 74, N1-N13. doi $10.1190 / 1.3008544$

Rubino, J. G., Velis, D. R., \& Sacchi, M. D. (2011). Numerical analysis of waveinduced fluid flow effects on seismic data: Application to monitoring of $\mathrm{CO}_{2}$ storage at the Sleipner field. J. Geophys. Res., 116, 1088-1102. doi: 10.1029/2010JB007997

Shi, J., Xuw, Z., \& Durucan, S. (2011). Supercritical $\mathrm{CO}_{2}$ core flooding and imbibition in Tako sandstone- Influence of sub-core scale heterogeneity. Int. J. Greenh. Gas Con., 5, 75-87. doi: 10.1016/j.ijggc.2010.07.003

Solazzi, S. G. (2018). Modelado y análisis de la respuesta sísmica de rocas heterogéneas saturadas por fluidos inmiscibles (PhD thesis, Facultad de Ciencias Astronómicas y Geofísicas, Universidad Nacional de La Plata). Retrieved from SEDICI. (http://sedici.unlp.edu.ar/handle/10915/67336).

Soldi, M., Guarracino, L., \& Jougnot, D. (2017). A simple hysteretic constitutive model for unsaturated flow. Transport Porous Med., 120(2), 271-285.

Subramaniyan, S., Quintal, B., Madonna, C., \& Saenger, E. H. (2015). Laboratorybased seismic attenuation in fontainebleau sandstone: Evidence of squirt flow. J. Geophys. Res. Solid Earth, 120(11), 7526-7535.

Teja, A. S., \& Rice, P. (1981). Generalized corresponding states method for viscosi- 
ties of liquid mixtures. Ind. Eng. Chem. Fundam., 20, 77-81.

Timur, A. (1968). An investigation of permeability, porosity, and residual water saturation relationships. In SPWLA 9th Annual Logging Symposium.

Tisato, N., \& Madonna, C. (2012). Attenuation at low seismic frequencies in partially saturated rocks: Measurements and description of a new apparatus. $J$. Appl, Geophys., 86, $44-53$.

Tisato, N., \& Quintal, B. (2013). Measurements of seismic attenuation and transient fluid pressure in partially saturated Berea sandstone: Evidence of fluid flow on the mesoscopic scale. Geophys. J. Int., 195, 342-351. doi: 10.1093/gji/ggt259

Toms-Stewart, J., Müller, T. M., Gurevich, B., \& Paterson, L. (2009). Statistical characterization of gas-patch distributions in partially saturated rocks. Geophysics, 72(2), WA51-WA64.

Tronicke, J., \& Holliger, K. (2005). Quantitative integration of hydrogeophysical data: Conditional geostatistical simulation for characterizing heterogeneous alluvial aquifers. Geophysics, 70, H1-H10. doi: 10.1190/1.1925744

Tyler, S. W., \& Wheatcraft, S. W. (1990). Fractal processes in soil water retention. Water Resour. Res., 26(5), 1047-1054.

van Genuchten, M. T. (1980). A closed-form equation for predicting the hydraulic conductivity fo unsaturated soils. Soil Sci. Soc. Am. J., 44, 892-898.

Vargaftik, N. B., Volkov, B. N., \& Voljak, L. D. (1983). International tables of the surface tension of water. J. Phys. Chem. Ref. Data, 12(3), 817-820.

White, J. E. (1975). Computed seismic speeds and attenuation in rocks with partial gas saturation. Geophysics, 40, 224-232. doi: 10.1190/1.1440520

Wood, A. (1955). A Textbook of Sound. New York: MacMillan Publishing Company.

Xu, P. (2015). A discussion on fractal models for transport physics of porous media. Fractals, 23(03), 1530001.

Yin, C. S., Batzle, M. L., \& Smith, B. J. (1992). Effects of partial liquid/gas saturation on extensional wave attenuation in Berea sandstone. Geophys. Res. Lett., 19(13), 1399-1402.

Yu, B., Li, J., Li, Z., \& Zou, M. (2003). Permeabilities of unsaturated fractal porous media. Int. J. Multiph. Flow, 29(10), 1625-1642.

Zhang, Y., Nishizawa, O., Kiyama, T., \& Xue, Z. (2015). Saturation-path de- 
pendency of P-wave velocity and attenuation in sandstone saturated with $\mathrm{CO}_{2}$ and brine revealed by simultaneous measurements of waveforms and X-ray computed tomography images. Geophysics, 80, D403-D415.

doi: 10.1190/GEO2014-0289.1 